\title{
CULTURA Y NATURALEZA COMO ELEMENTOS PARA EL DESARROLLO DEL ECOTURISMO EN MATAHUASI- CONCEPCIÓN*
}

\author{
Maldonado Oré, Edith Mercedes y y Ortíz Espinar, Manuel Adolfo²
}

Facultad de Antropología de la Universidad Nacional del Centro del Perú

\begin{abstract}
RESUMEN
El ecoturismo se muestra como una opción factible para la conservación del patrimonio cultural y natural de muchas regiones y localidades del país que buscan un equilibrio sustentable. Los conocimientos tradicionales sobre tecnologías agrícolas (manejo de suelos, agua, semillas, pisos ecológicos), ganaderas, plantas medicinales, gastronomía, música, danzas, festividades, tradiciones orales etc. que representan la cultura viva del pueblo del distrito de Matahuasi y que son trasmitidos de generación en generación pueden ayudar a enfrentar los retos de su supervivencia en un mundo en constante cambio como el que se vive hoy. En función a los recursos culturales y naturales en Matahuasi, se puede desarrollar las siguientes formas de turismo: el senderismo, el turismo de paisajes, la observación de flora y fauna, la recreación, la educación ambiental, el agroturismo, el turismo vivencial y la gastronomía.
\end{abstract}

Palabras clave: senderismo, turismo vivencial, agroturismo, turismo cultural, gastronomía, turismo de paisajes.

\section{CULTURE AND NATURE AS ELEMENTS FOR THE DEVELOPMENT OF ECOTOURISM IN MATAHUASI-CONCEPCION}

\begin{abstract}
The ecoturism is like a feasible option for the conservation of cultural and natural patrimony of many regions and localities of the country that look for a sustainable balance. The traditional knowledge on agricultural technologies (ground handling, water, seeds, ecological floors), cattle, medicinal plants, gastronomy, music, dances, festivity, oral traditions. Which represent the alive culture of the town of Matahuasi district and are transmited from generation to generation can help to face the challenges of its survival in a constant change world as it is lived today. In Function to the cultural and natural resources in Matahuasi, it is possible to develope the following tourism forms: The senderism, the landscapes tourism, the flora and fauna observation, the recreation, the environmental education, agroturism, existential tourism and gastronomy.
\end{abstract}

Key words: senderism, existential tourism, agroturism, cultural tourism, gastronomy, tourism of landscapes.

\footnotetext{
* Este trabajo de investigación fue recibido el 20/12/2008 retornado para su revisión 26/10/2009 y aprobado para su publicación 16/11/2009

1Email: edmaore@yahoo.es

22Email: manuelortize@hotmail.com
} 


\section{INTRODUCCIÓN}

En esta investigación se planteó el tema de la cultura y la naturaleza como elementos para el desarrollo del ecoturismo teniendo en consideración que la diversidad cultural y natural del distrito de Matahuasi generaría el interés de personas en conocer la interrelación hombre naturaleza y cultura. El estudio también permite clarificar la identidad local, rescatando la historia natural y cultural, lo que permitirá generar actividades en base a ellas, relacionadas con el turismo.

La utilización de la cultura, como atracción para promover el desarrollo del turismo, se ha hecho cada vez más evidente. Si primero fueron los centros históricos, los sitios arqueológicos y los museos bien provistos, ahora las fiestas, las ceremonias y rituales, las prácticas cotidianas como cocina, artesanía y otros usos simbólicos son movilizados como recursos en el turismo y en la promoción de industrias centradas en el aprovechamiento del patrimonio cultural y natural.

Los objetivos planteados para la presente investigación fueron:

\section{General:}

Identificar los recursos culturales y naturales potenciales, en la relación hombre - naturaleza en Matahuasi para fines del ecoturismo.

\section{Específicos:}

- Identificar costumbres, ritos y tradiciones culturales que demuestren la relación hombre naturaleza y cultura.

- Identificar los recursos artístico - culturales (festividades, danzas, etc.) y gastronomía.

\section{MATERIALES Y MÉTODOS}

\section{MATERIALES}

\section{LUGAR DE ESTUDIO}

Matahuasi deriva de las palabras quechuas: mata $=$ pegado y huasi $=$ Casa, lo que significa casas pegadas. a. Ubicación

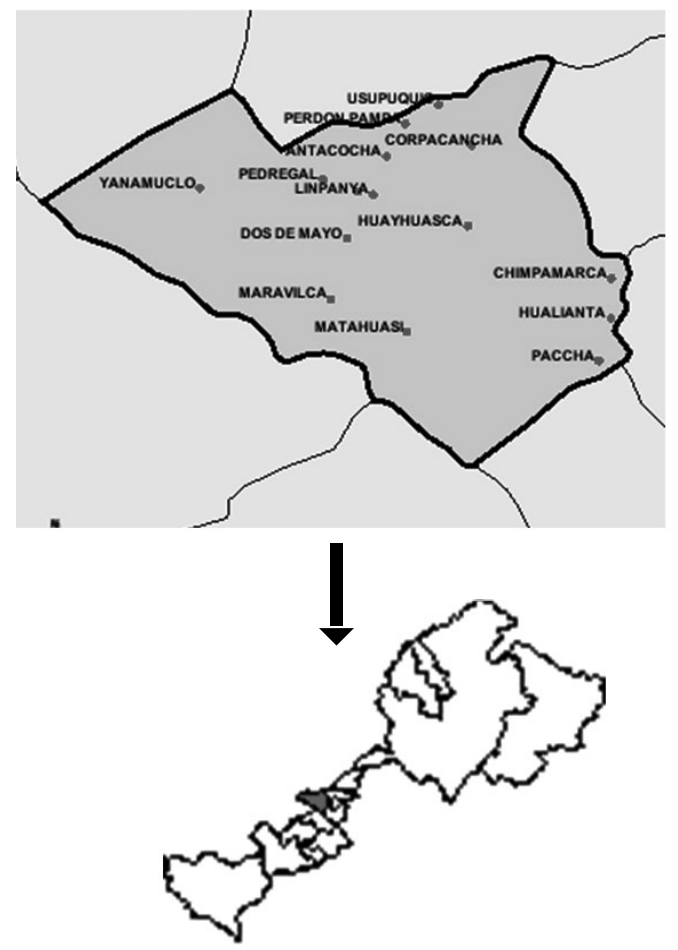

Figura 1. Mapa de ubicación del distrito de Matahuasi.

El distrito de Matahuasi se encuentra ubicado en la margen izquierda del valle al noreste de la provincia de Concepción. Geográficamente se encuentra ubicado a $11^{\circ} 53^{\prime} 37^{\prime \prime}$ latitud sur y $75^{\circ} 20^{\prime} 37^{\prime \prime}$ longitud oeste.Pertenece al piso ecológico quechua.

Se encuentra en la zona de vida bosque seco-montano bajo tropical (bs-MBT). Es atravesado por tres ríos: Mantaro, Achamayo y Seco. Cuenta con una laguna con presencia de avifauna migratoria y permanente.

Limita por el norte con el distrito de Apata (provincia de Jauja); por el este con el distrito de Santa Rosa de Ocopa y el distrito de Nueve de Julio, por el sur con el distrito de Concepción y por el oeste con el río Mantaro. 


\section{METODOLOGÍA}

Para la recopilación de los datos de campo se elaboró materiales como las guías de entrevista y los cuestionarios, fichas de levantamiento de datos culturales, naturales y otros. También se usó equipos audiovisuales para registrar en forma gráfica los recursos culturales y naturales. La investigación es de índole cualitativa cuantitativa.

En la fase de campo se obtuvo datos de fuentes primarias y son aquellos que se tomaron del contacto directo con la realidad empírica. Las técnicas usadas fueron la observación (directa y participante), la entrevista y el cuestionario para captar la realidad de cada ámbito de estudio y el registro etnográfico para obtener los datos culturales de las actividades de la población. Se realizó el recojo de datos in situ en los cuatro distritos. Las muestras se eligieron según la actividad de cada distrito.

\section{Entrevistas}

Fueron aplicadas a las autoridades locales y a la muestra elegida para conocer qué tipo de recursos habían inventariado y al tipo de turismo que se orientaban.

\section{Registro etnográfico}

Se aplicaron a los productores agropecuarios: dueños de establos, productores de queso artesanal, que permitió la obtención de datos de los aspectos culturales tradicionales de éstas actividades que pueden ser usados como elementos integradores para el desarrollo del ecoturismo.

\section{RESULTADOS}

\section{RECURSOS, CULTURA Y ECOTURISMO EN EL DIS- TRITO DE MATAHUASI}

El distrito de Matahuasi no está considerado dentro del circuito de turismo convencional del Valle del Mantaro, ni de la provincia de Concepción, debido a que no se había identificado ningún recurso válido que pueda ser usado para el turismo.

Con la investigación se logró identificar varios recursos de uso potencial para el ecoturismo (ver cuadro 1).
Cuadro 1. Recursos potenciales de Matahuasi, sus valores culturales y naturales.

Cuadro 1. Recursos potenciales de Matahuasi, sus valores culturales y naturales.

\begin{tabular}{|c|c|c|c|}
\hline RECURSO & VALOR NATURAL & $\begin{array}{c}\text { VALOR } \\
\text { CULTURAL }\end{array}$ & $\begin{array}{c}\text { TIPOS DE } \\
\text { ECOTURISMO }\end{array}$ \\
\hline $\begin{array}{l}\text { Qhapaq Ñan } \\
\text { (camino Inca, } \\
\text { camino real) }\end{array}$ & $\begin{array}{l}\text { Interrelación de flora } \\
\text { y fauna } \\
\text { Fuentes de agua } \\
\text { Tierras de cultivo } \\
\text { Conservación de } \\
\text { especies }\end{array}$ & $\begin{array}{l}\text { Histórico } \\
\text { Actividades } \\
\text { económicas de la } \\
\text { población } \\
\text { Conocimiento de } \\
\text { las propiedades } \\
\text { medicinales de } \\
\text { las plantas } \\
\text { Uso alimenticio, } \\
\text { ritual y } \\
\text { económico de la } \\
\text { flora y fauna }\end{array}$ & $\begin{array}{l}\text { Senderismo } \\
\text { Turismo de } \\
\text { paisajes } \\
\text { Recreación } \\
\text { Observación de } \\
\text { flora y fauna }\end{array}$ \\
\hline $\begin{array}{l}\text { Humedal "La } \\
\text { Pampa" }\end{array}$ & $\begin{array}{l}\text { Avifauna } \\
\text { Ictiofauna } \\
\text { Vegetación } \\
\text { Interrelación de las } \\
\text { especies en el } \\
\text { ecosistema } \\
\text { Agua }\end{array}$ & $\begin{array}{l}\text { Histórico } \\
\text { Creencias } \\
\text { Costumbres } \\
\text { Folclore }\end{array}$ & $\begin{array}{l}\text { Observación de } \\
\text { avifauna } \\
\text { Turismo de } \\
\text { paisajes } \\
\text { Recreación } \\
\text { Educación } \\
\text { ambiental }\end{array}$ \\
\hline
\end{tabular}

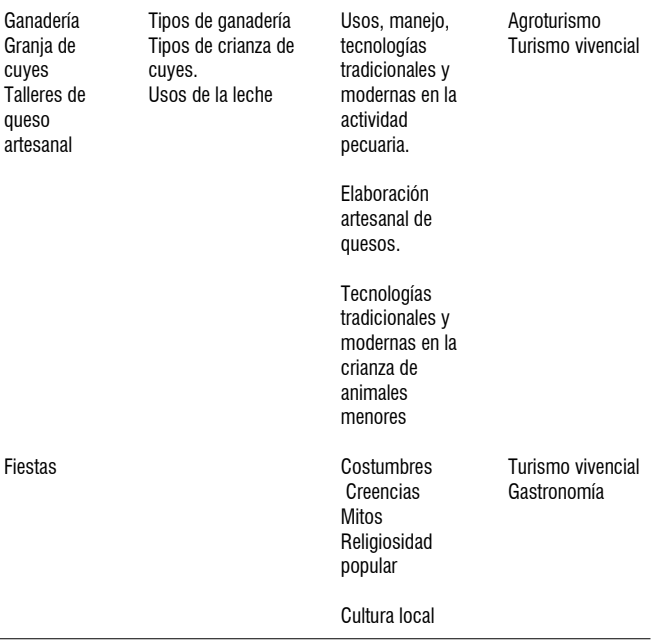

Fuente: Elaboración propia de los investigadores.

\section{DISCUSIONES}

Los resultados obtenidos que se muestran en el cuadro 1, nos permite realizar el análisis y la discusión de cada uno de los recursos culturales y naturales así como el tipo de ecoturismo que se puede realizar en cada uno de ellos. 


\section{Qhapaq Ñan (Camino Inca o "Real")}

El Qhapaq Ñan o camino inca es conocido como camino "real" en el Valle del Mantaro. Atraviesa casi todos los distritos del valle como es el caso de Concepción y Matahuasi: el tramo de Matahuasi se encuentra más íntegro y en él se observa flora nativa y exótica, avifauna silvestre, fuentes de agua y otros recursos naturales. También hay manifestaciones culturales de la vida cotidiana de la población local: sus viviendas tradicionales, agricultura, ganadería y comercio que servirían de insumo para usarlo en la práctica del senderismo y el turismo de paisajes. La longitud es de $3.5 \mathrm{~km}$ partiendo desde el humedal "La Pampa" hasta el paraje San Juan en el anexo de Yanamuclo. Este camino permite establecer los usos y aprovechamientos en la dinámica de la población local.

Se muestran las características del Qhapaq Ñan a través de las siguientes fotografías:
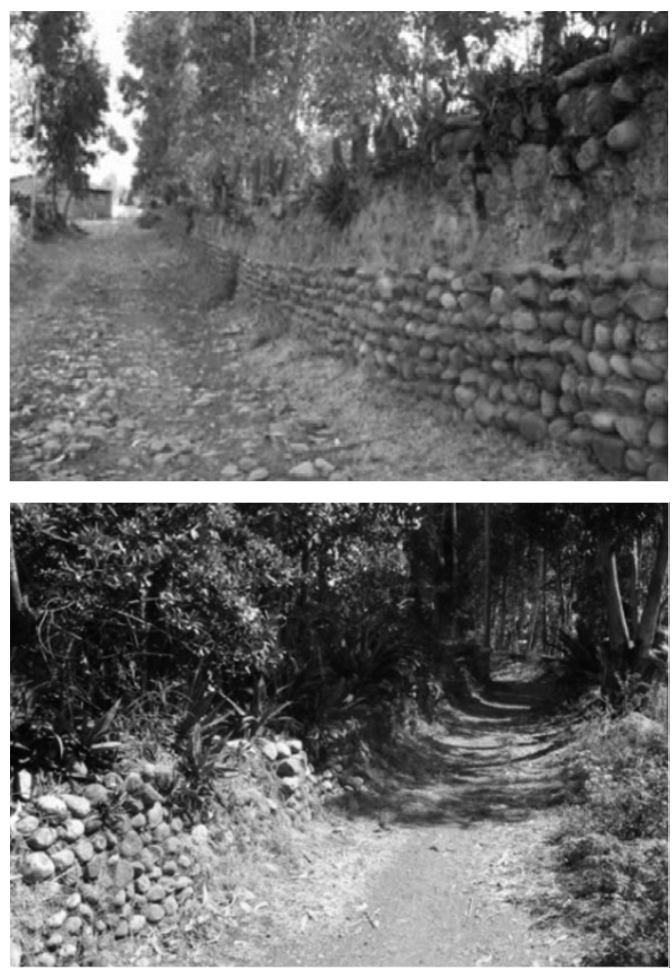

Foto 1 y 2. El Qhapaq Ñan con vestigios culturales de la construcción de sus paredes con cantos rodados, característica que se conserva en tramos del camino.
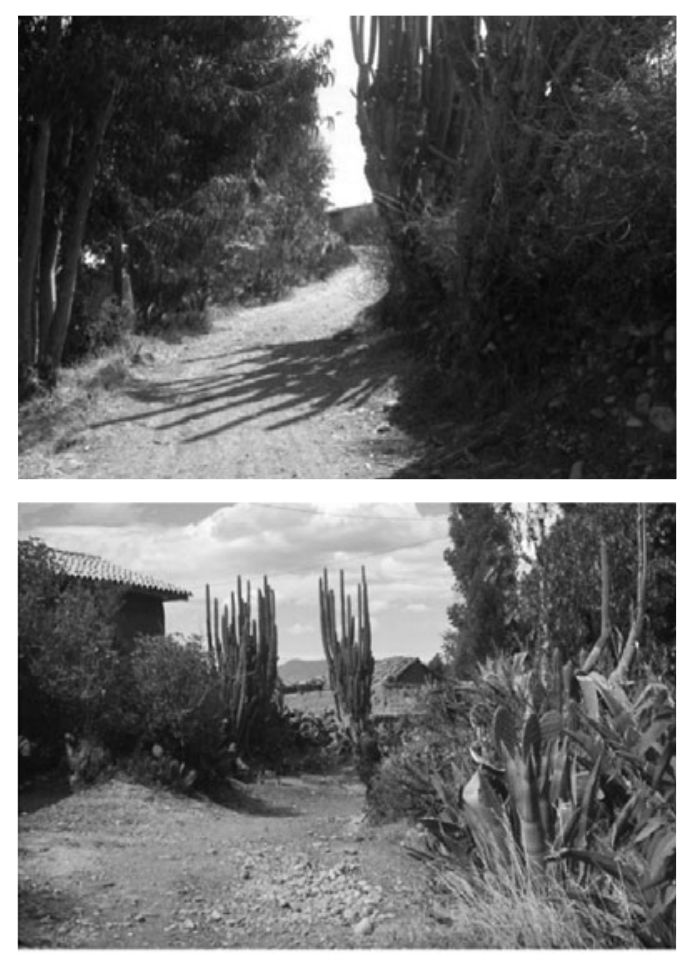

Foto 3 y 4. El camino rodeado de especies que sirven para la protección de cultivos: eucaliptos, cactus y maguey.

\section{Senderismo en el Qhapaq Ñan}

El senderismo en el Qhapaq Ñan surge como una propuesta de recuperación de una actividad innata al medio rural como es el hecho de caminar utilizando aquellas vías y senderos, que durante décadas han permitido la relación de la población rural con su entorno más inmediato y con el resto del territorio, pero con un concepto mucho más amplio y rico del sendero que entiende a éste ya no sólo como forma de aproximación sino también, como forma de contacto y conocimiento del medio natural y cultural de las zonas rurales.

En el trayecto se identificó especies de avifauna y flora que tienen mucho valor en el equilibrio del ecosistema, en la cultura y vida diaria de la población. En cuanto a la flora se identificaron las especies más representativas de árboles y arbustos nativos e introducidos que tienen mucha importancia cultural, ritual y económica para la población local. Muchas de estas especies son usadas por sus propiedades medicinales, alimenticias, artesanales, como leña y como material de construcción en sus viviendas. 
La práctica del senderismo, tendría objetivos educativos, recreativos y deportivos, pretendiéndose con él, disfrutar de interesantes paisajes y valorar el patrimonio natural, histórico y cultural que ofrece. También se buscaría recuperar el paisaje para la persona y reencontrarla con el sistema de vida tradicional. Se devolvería al camino el concepto de lugar de encuentro y de intercambio que tuvo a lo largo de la historia.

El senderismo en el Qhapaq Ñan promovería la conservación del ecosistema natural y contribuiría con la mejora de las economías locales al ofrecer al visitante una experiencia educativa ambiental basada en la naturaleza manejada de manera sostenible.

\section{Observación de avifauna en el humedal "La Pampa"}

El humedal "La Pampa" del Distrito de Matahuasi es pequeño en comparación con otros que existen en la costa y sierra del país y que tienen mayor biodiversidad. Este humedal puede resultar importante para la observación de la avifauna silvestre. También se identificaron las especies más representativas que se encuentran en él.
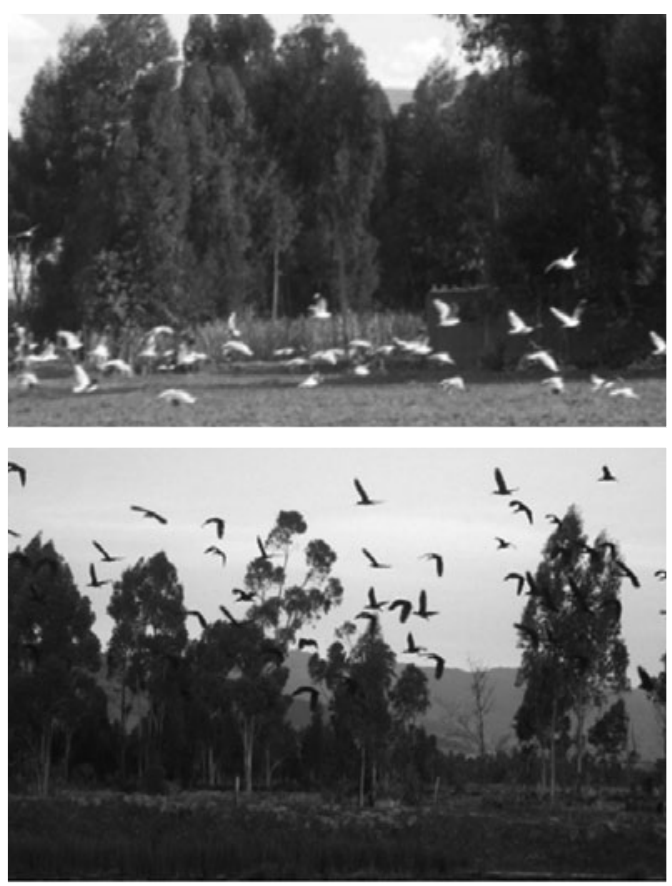

Fotos 5 y 6. Observación de avifauna silvestre en el humedal La Pampa: gaviotas y yanavicos.
Para que el humedal "La Pampa" pueda ser usado para el ecoturismo en su interrelación con la cultura y la naturaleza, en primer lugar habría que poner en valor el sitio, tal vez con una propuesta municipal de declarar la zona como refugio municipal o comunal de avifauna silvestre. Esto podría ser una estrategia que permita proteger el humedal y conservar la diversidad biológica y luego manejarlo para un posible uso en diversas formas de turismo ligado a la naturaleza y la cultura local.

La educación ambiental en base a la observación de aves y su debida interpretación, permitińa realizar una actividad pedagógica dirigida a los ecoturistas que revelaría el significado y las relaciones naturales de las aves mediante el uso de objetos originales y por medios ilustrativos más que mediante la simple comunicación de información logrando de esta manera que se descubra y comprenda que el hombre es también una especie de la naturaleza que está contemplando.

\section{Paisaje}

Los paisajes culturales representan las obras que "combinan el trabajo del hombre y la naturaleza”, de acuerdo al Artículo 1 de la Convención sobre Patrimonio Mundial. El término «paisaje cultural» incluye una diversidad de manifestaciones de la interacción entre el hombre y su ambiente natural.

Los paisajes andinos, son ahora predominantemente agropecuarios. En Matahuasi encontramos paisajes cuyos elementos son los campos de cultivo, las viviendas tradicionales, actividades cotidianas del poblador, la flora y fauna exótica y nativa, caminos, paisajes del amanecer y atardecer en la campiña y la agroforestería.

Los paisajes que pueden ser usados para el turismo de paisajes se pueden encontrar predominantemente en las campiñas alrededor del Qhapaq Ñan y del humedal La Pampa.

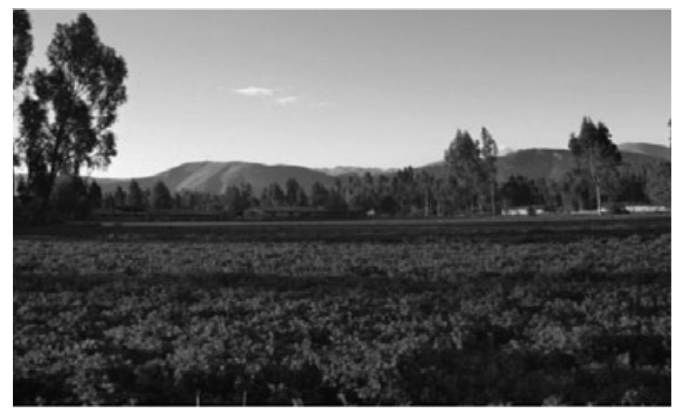

Foto 7. Turismo de paisajes. Paisaje y agroforestería 
Si bien los paisajes presentados no entran en la clasificación de la categoría de paisaje cultural de la UNESCO, sin embargo sirven para fomentar el turismo de paisajes y la educación ambiental, ya que se les considera como parte del patrimonio natural y cultural de esta localidad.

\section{Fiestas tradicionales}

Las fiestas en Matahuasi, como en otros lugares del Valle del Mantaro, son construcciones míticas y simbólicas en las que se manifiestan creencias, mitos, concepciones de la vida y del mundo y que están asociadas a algunas etapas del ciclo vital, la fe religiosa y otras motivaciones humanas. Se transmiten por tradición y son originales y propias de ésta sociedad en un espacio y un tiempo determinados.

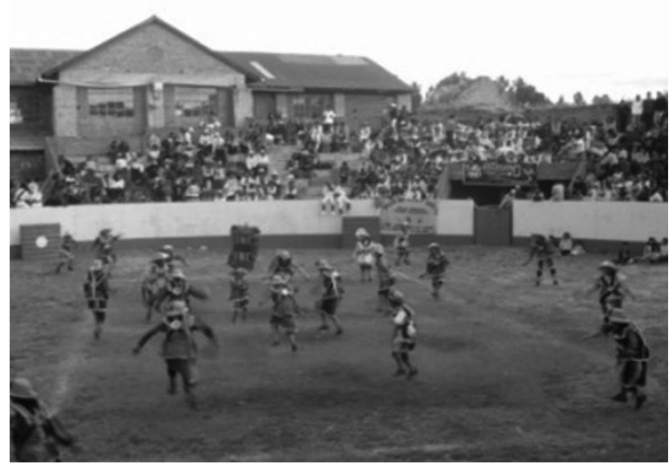

Foto 8. Danzas para el turismo vivencial

El uso de las fiestas como un recurso para el turismo permitiría el fortalecimiento de la identidad cultural de los pobladores de Matahuasi y el reconocimiento de la diversidad mediante la apropiación social del patrimonio cultural. En la medida en que la gestión pública, los instrumentos normativos y las acciones administrativas consideren los valores y el significado de las fiestas se puede lograr la sostenibilidad en cuanto a su estructura, los elementos que utilizan y los recursos que se requieren.

Uno de los principales pasos para integrar la cultura con la actividad turística, y que éstas se conviertan en herramientas de desarrollo, es fortalecer la identidad cultural de cada pueblo a través de la educación, tomando conciencia sobre la importancia del legado cultural.

\section{Agroturismo}

El agroturismo está concebido para complementar la actividad agropecuaria, pero no para sustituirla. Está dirigido para viajeros procedentes de zonas urbanas que buscan algo diferente, como un encuentro con la naturaleza y las manifestaciones culturales.

En Matahuasi sobresalen tres actividades que pueden ser recursos importantes para el agroturismo: crianza de ganado vacuno, elaboración de queso artesanal y crianza de cuyes.
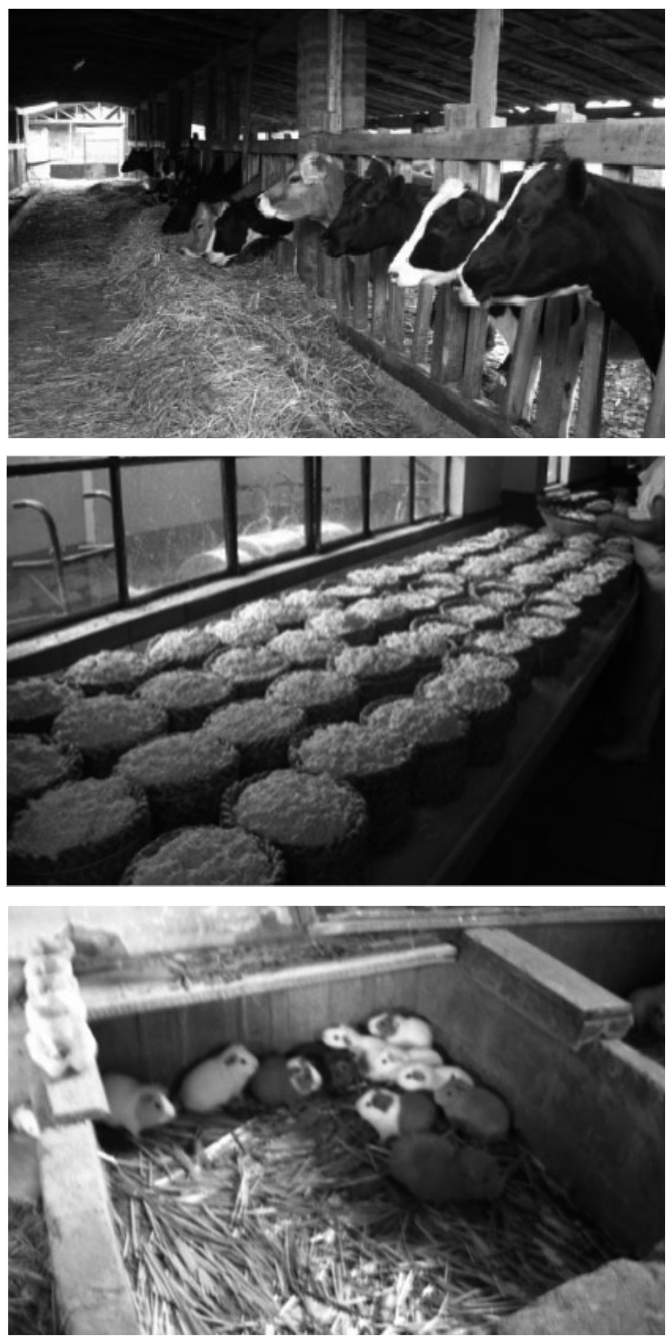

Foto 9, 10 y 11. Recursos agropecuarios para el desarrollo del agroturismo. 
Las tres actividades son recursos potenciales para el desarrollo del agroturismo que es una forma de turismo rural comunitario. Esta actividad se puede integrar a la economía local y a las actividades propias de este medio.

Para que esta forma de turismo se constituya en una actividad sostenible es necesario capacitar, concienciar y educar a las familias ganaderas, productoras de queso y criadoras de cuyes en cuanto se refiere a la capacidad de interactuar con los visitantes, mejorar sus infraestructuras de vivienda y de servicios para compartirlos con los turistas.

\section{CONCLUSIONES}

- El patrimonio cultural tangible e intangible y el patrimonio natural es la mayor riqueza con que cuenta este distrito y constituye la base de su identidad, son recursos de importancia fundamental y que pueden convertirse en la piedra angular para lograr su bienestar y desarrollo a través del ecoturismo.

- En el tramo del Qhapaq Ñan ubicado en Matahuasi, se puede desarrollar el senderismo, el turismo de paisajes, la observación de flora y fauna y la recreación.

- En el Humedal "La Pampa" se puede desarrollar la observación de avifauna, el turismo de paisaje y la educación ambiental.

- En los establos ganaderos, granjas de cuyes y talleres de elaboración artesanal de queso, el agroturismo y turismo vivencial.

- En las fiestas patronales, el turismo vivencial y la gastronomía.

- La propuesta de desarrollo del ecoturismo con base en los atractivos naturales y las manifestaciones culturales permitiría desarrollar a la población local y mejorar la calidad de vida de los pobladores buscando la sostenibilidad económica.

\section{LITERATURA CITADA}

Instituto Nacional de Estadística e Informática. 2006. Clasificación nacional de actividades económi- cas del Perú. ClaNAE-Perú. Dirección Nacional de Cuentas Nacionales 429 pg. Consultado 12 de julio 2007. Disponible en http://iinei.inei.gob. pe/ftp/CLANAE2006.pdf.

Ministerio de Comercio Exterior y Turismo. 2007. “Promo Artesanía 2007."Articulación TurismoArtesanía y las industrias culturales locales. 2404-2007. Disponible en: http://www.arteturcites. com.pe/ noticias.php?noti=141.

Ministerio de Comercio Exterior y Turismo. 2007. Lineamientos para el desarrollo del turismo rural comunitario en el Perú. Viceministerio de Turismo. Dirección de Desarrollo de Producto Turístico. 61 pg. Consultado 20 de abril 2007. Disponible en: http://www.mincetur.gob.pe/TURISMO/ LEGAL/LINEAMIENTOS_TURISMO_RURAL.pdf.

Organización de las Naciones Unidas para la Educación, la Ciencia y la Cultura. 2004. Patrimonio cultural intangible: nuevos planteamientos respecto a su salvaguardia. Departamento de Patrimonio Intangible. Consultado: 10 de marzo del 2007. Disponible en: http://www.crim.unam. $\mathrm{mx} /$ cultura/informe/ informe\%20mund2/PATRIMONIO .htm

Organización Mundial del Turismo. 1999. Agenda para planificadores locales: Turismo sostenible y gestión municipal. Edición para América Latina y el Caribe. Una publicación de turismo y medio ambiente. The British Council. 156 pg.

Riveros, H ; Blanco, M. 2003. El agroturismo, una alternativa para revalorizar la agroindustria rural como mecanismo de desarrollo local. Programa de Desarrollo de la Agroindustria Rural para América Latina y el Caribe. Perú. 33 pg. 